\title{
III. - ÉVOLUTION DE LA MICROFLORE
}

\author{
Jeanne POISSON \\ avec la collaboration technique de B. Cahagnier et C. Desserme \\ Station de Biochimie et Physicochimie des Céréales, \\ Section Microbiologie, I. N. R. A., \\ 16, rue Nicolas-Fortin, \\ Paris (13e) \\ RÉSUMÉ
}

Les examens microbiologiques effectués au cours des essais ont permis de vérifier l'efficacité des traitements de séchage, de contrôler la stabilité des lots pendant le stockage et d'apprécier la qualité hygiénique du grain destiné à l'expérimentation sur animaux. Les meilleurs lots ont été obtenus par séchage immédiat après la récolte.

\section{INTRODUCTION}

L'examen des caractéristiques quantitatives et qualitatives de la microflore des grains permet, dans une certaine mesure, d'apprécier leur qualité et leur degré d'appropriation à une bonne conservation, de juger de 1'efficacité de certains traitements qui leur sont appliqués et de contrôler les opérations de stockage (CHrisTENSEN et KAUFMANN, I965; MULTON et al., I969).

En effet, la microflore des céréales, par sa seule présence, par son éventuel développement et les produits de son métabolisme, est susceptible de contribuer à la diminution de la valeur technologique, nutritionnelle et hygiénique du grain.

C'est ainsi qu'elle peut entraîner une perte pondérale et un changement d'aspect des lots envahis, qu'elle modifie la valeur nutritive de l'aliment, sa flaveur et son appétibilité par la disparition de certains composants du grain ou la synthèse de principes indésirables. De plus, une microflore trop abondante dans les produits ingérés peut provoquer des troubles organiques et jouer le rôle d'allergène. Enfin, la multiplication d'une flore pathogène et toxigène est toujours à redouter (SPICHER, I962; SEMENIUK, I954; MOREAU, I960). 
Il est donc important de réduire au maximum et le plus tôt possible la microflore des grains et d'empêcher la multiplication de la microflore résiduelle.

Les opérations de séchage réalisées dans le cadre de la présente expérimentation, en abaissant la teneur en eau des grains de 35-36 p. Ioo à environ I 3 p. Ioo les ont mis à 1'abri d'une attaque bactérienne et d'une multiplication des levures et moisissures les plus courantes, seul restant à craindre un très lent développement de quelques variétés de moisissures qui parviennent à germer à une humidité relative de $62,5 \mathrm{p}$. Ioo (valeur qui, pour le maïs, correspond à une teneur en eau voisine de $\mathrm{I} 3 \mathrm{p}$. Ioo à $22^{\circ} \mathrm{C}$ ).

Dans ces conditions, le but des examens microbiologiques effectués au cours des essais a été de vérifier l'efficacité du séchage, mais surtout de contrôler la stabilité microbiologique des lots séchés au cours de la période de stockage qui a précédé leur utilisation et d'évaluer leur degré de pollution à ce moment.

\section{MATÉRIEL E'T MÉTHODES}

- Les analyses bactériologiques ont été effectuées sur le témoin de récolte (Lot TTR), sur les lots placés sous ventilation d’air refroidi avant séchage et sur les lots séchés destinés à l'alimentation animale.

- Le dénombrement de la population microbienne du maïs est effectué selon le protocole suivant (Poisson et al., 1964) :

Un échantillon moyen de $5^{\circ} \mathrm{g}$ de grain est broyé aseptiquement dans $400 \mathrm{ml}$ de diluant (peptone : I $\mathrm{g}$; chlorure de sodium : $9 \mathrm{~g}$; Tween $80: 0,03 \mathrm{~g}$; eau distillée sur verre : $\mathrm{q}$. $\mathrm{s}$. pour $\mathrm{I}$ ooo $\mathrm{ml}$ ), pendant go secondes à une vitesse de $\mathrm{r} 2$ ooo $\mathrm{t} / \mathrm{mn}$.

Les microorganismes de la suspension obtenue sont dénombrés suivant la méthode classique des dilutions décimales et de culture en boîtes de Pétri sur milieux nutritifs.

L'originalité du procédé réside dans l'importance de la prise d'essai qui doit pallier la grande hétérogénéité de la répartition des microorganismes sur les grains.

Les bactéries mésophiles aérobies et aérobies-anaérobies facultatives, désignées par " bactéries totales " dans le tableau des résultats, sont cultivées à $30^{\circ} \mathrm{C}$ sur un milieu gélosé à base de malt-peptone (extrait de malt : $20 \mathrm{~g}$; peptone : $5 \mathrm{~g}$; gélose : I5 $\mathrm{g}$; eau distillée sur verre $\mathrm{q}$. $\mathrm{s}$. pour I ooo ml) et sur " gélose-numération " de l'Institut Pasteur.

Les bactéries coliformes sont cultivées sur une Gélose au désoxycholate (273 Difco).

Les moisissures sont cultivées à $22^{\circ} \mathrm{C}$ sur milieu malt gélosé (extrait de malt : $20 \mathrm{~g}$; gélose : I5 $\mathrm{g}$; eau distillée : $\mathrm{q}$. $\mathrm{s}$. pour I ooo $\mathrm{ml} ; \mathrm{pH}$ 7) et sur milieu Czapek, en présence de Pénicilline et de Streptomycine (Porsson, I967).

\section{RÉSULTATS ET INTERPRÉTATION DES RÉSULTATS}

Les résultats obtenus sont rassemblés dans le tableau I et illustrés par les figures $x$ et 2 ; bien que leur interprétation soit rendue délicate par la répartition toujours très hétérogène des microorganismes sur les grains, il a été possible d'observer :

I $^{0}$ Une action dépressive nette des traitements thermiques sur la population bactérienne des lots. Celle-ci est réduite d'environ 6 fois dans le cas du maîs traité aussitôt après la récolte, et de plus de 40 fois dans le cas des lots séchés après conservation par ventilation d'air refroidi. 


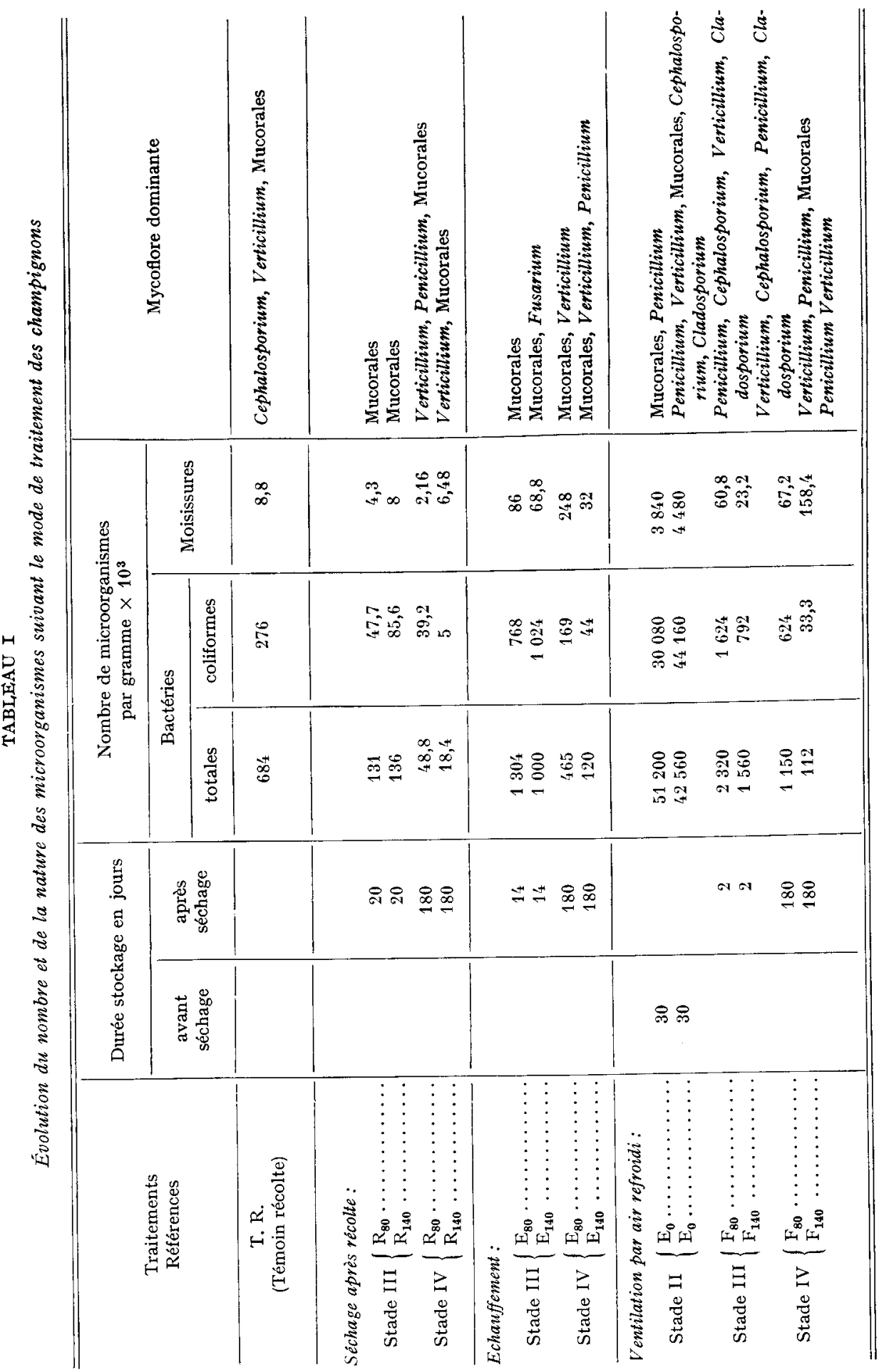


Les populations bactériennes des lots séchés à $80^{\circ} \mathrm{C}$ et à $\mathrm{I} 40^{\circ} \mathrm{C}$ sont pratiquement identiques (fig. I) : ceci est à rapprocher du fait que les températures de l'air à la sortie des séchoirs sont très voisines, et qu'il y a toujours une recontamination des grains par 1'air ambiant et les manipulations après traitement. Cependant, à long terme, en fin de stockage, 1'action dépressive du séchage à $\mathrm{I} 40^{\circ} \mathrm{C}$ paraît un peu plus importante qu'à $80^{\circ} \mathrm{C}$.

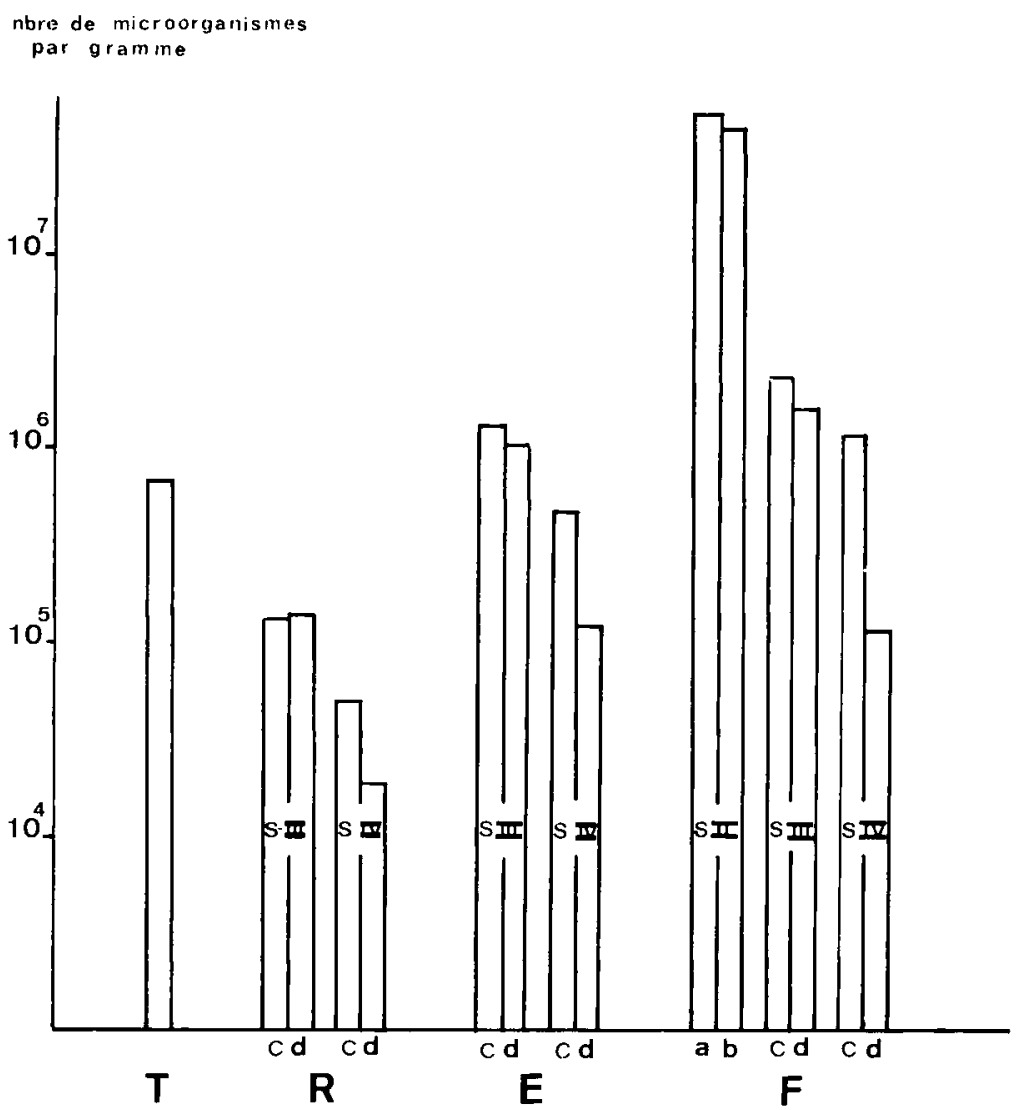

FIG. I. - Importance de la population bactérienne des lots en fonction des traitements effectués.

$$
\begin{aligned}
& \mathrm{T}=\text { Témoin } \\
& \mathrm{R}=\text { Récolte } \\
& \mathrm{E}=\text { Echauffé } \\
& \mathrm{F}=\text { Ventilation par air refroidi }
\end{aligned}
$$

- en abscisse sont indiqués les traitements ( $\mathrm{T}$ : témoin de récolte, $\mathrm{R}$, et $\mathrm{F}$ ) et les stades (S) :

$a=$ avant séchage à $80^{\circ} \mathrm{C}$

$b=$ avant séchage à $140^{\circ} \mathrm{C}$

$c=$ après séchage à $80^{\circ} \mathrm{C}$

$d=$ après séchage à $140^{\circ} \mathrm{C}$

- en ordonnée est indiqué le nombre de microorganismes par gramme de grain.

En ce qui concerne les moisissures des lots séchés après récolte, il y a eu destruction d'une partie de la flore champêtre par le séchage, suivie d'une recontamination ; par contre, le séchage après conservation par ventilation d'air refroidi a eu une 
action dépressive très nette, sans qu'il y ait de différence significative entre les deux températures (fig. 2).

Les lots E n'ayant pas été examinés à la fin de la période de préstockage, il est difficile d'apprécier l'action du séchage dans cet essai. Mais au cours d'expériences antérieures avec du grain ayant une teneur en eau de 32 p. Ioo environ, il a été possible de mettre en évidence, pendant les 3 premiers jours de stockage (à $40 \mathrm{~cm}$ du sol) une augmentation des bactéries dont le nombre est multiplié par 38 , et une augmentation des moisissures dont le nombre est multiplié par I4; puis une régression

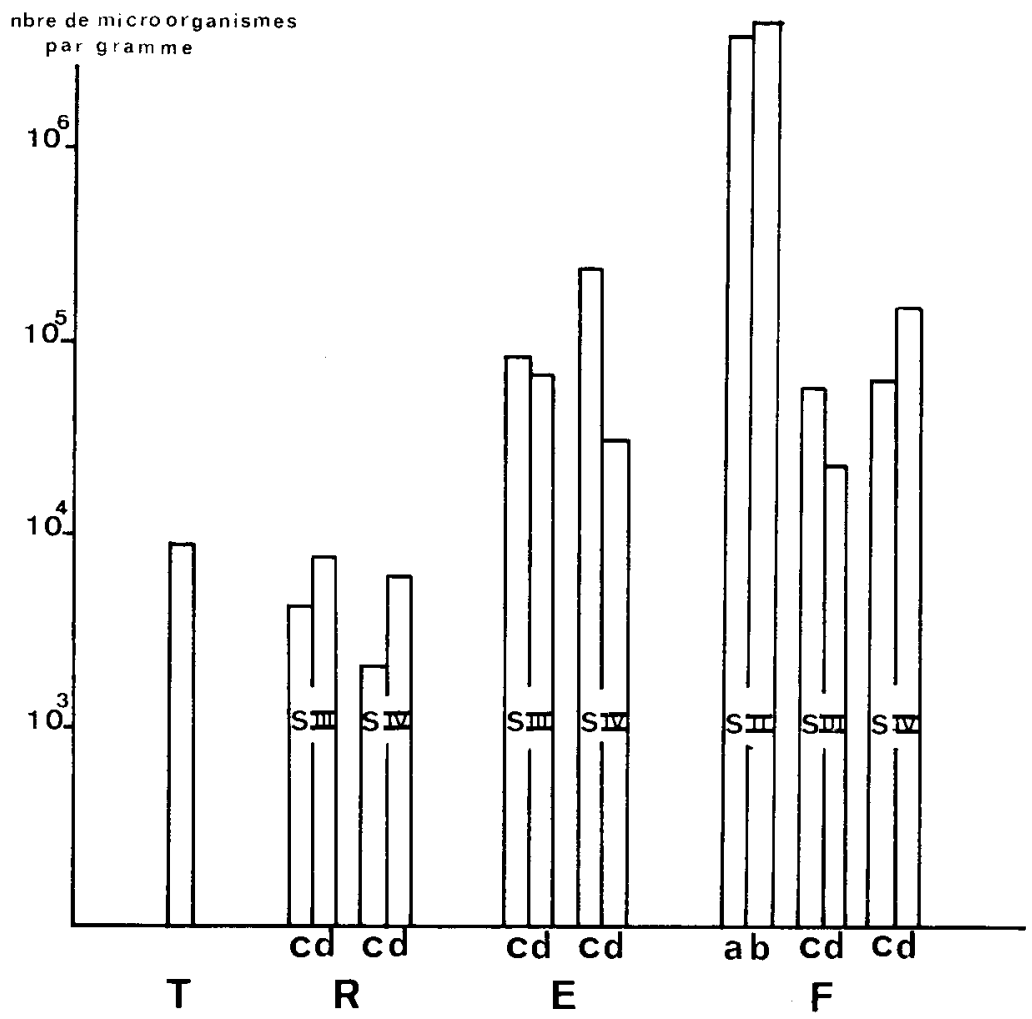

FIG. 2. - Importance de la population fongique des lots en fonction des traitements effectués :

- en abscisse sont indiqués les traitements ( $\mathrm{T}$ : témoin de récolte, $\mathrm{R}, \mathrm{E}$ et $\mathrm{F}$ ) et les stades (S) :

$a=$ avant séchage à $80^{\circ} \mathrm{C}$

$b=$ avant séchage à $140^{\circ} \mathrm{C}$

$c=$ après séchage à $80^{\circ} \mathrm{C}$

$d=$ après séchage à ${ }^{1} 40^{\circ} \mathrm{C}$

- en ordonnée est indiqué le nombre de microorganismes par gramme de grain.

des bactéries et des moisissures, alors que la température atteint $50^{\circ} \mathrm{C}$ environ. Quand la durée de l'essai se prolonge, les moisissures résiduelles se multiplient pendant la période de refroidissement quand la température est à nouveau favorable à leur développement. Un séchage à température élevée effectué à l'un ou l'autre de ces deux stades permet de réduire la population microbienne des grains.

$2^{\circ} L^{\prime}$ évolution de la microflore des lots séchés en cours de stockage :

- le nombre de bactéries a diminué dans tous les lots au cours du stockage, 
ce qui est normal, compte tenu des caractéristiques hydriques et thermiques réalisées ;

- le nombre de moisissures a diminué également dans tous les lots sauf dans les mais $\mathrm{E}_{80}$ et $\mathrm{F}_{140}$.

La diminution a surtout porté sur les Mucorales, ce qui a permis de mettre en évidence la flore champêtre résiduelle, et les Penicillium.

$\mathrm{L}_{\mathrm{a}}$ mycoflore du maïs $\mathrm{E}_{80}$, en très légère augmentation, révèle une contamination extérieure plutôt qu'une multiplication; par contre, il y a eu multiplication des Penicillium dans le $\mathrm{F}_{140}$, due vraisemblablement à la formation de quelques zones de condensation qui ont constitué des foyers d'infection.

$3^{\circ}$ La population microbienne des lots séchés destinés en fin de stockage à l'alimentation animale :

- A une exception près $\left(\mathrm{F}_{80}\right)$, le nombre de bactéries des grains stockés est inférieur ou approximativement égal à celui du témoin, la proportion de bactéries coliformes restant, dans l'ensemble, assez élevée comme dans le témoin.

- Dans les lots $\mathrm{R}$, le nombre de moisissures est très légèrement inférieur à celui du témoin. La mycoflore provient du champ de maïs et des contaminations, non d'une prolifération notable après récolte.

-- Dans les lots $\mathrm{E}, 1 \mathrm{a}$ mycoflore résiduelle après séchage et stockage est nettement plus importante que dans le témoin, mais elle est constituée par les mêmes espèces, à l'exception de celles qui sont thermosensibles; elle provient donc en majeure partie de la flore des champs et de la flore de contamination qui ont proliféré avant séchage. (Le stade II n'ayant pas été examiné, les renseignements sur la flore thermosensible qui a pu se développer au cours du préstockage manquent.)

- Dans les lots F, la mycoflore provient des espèces champêtres, qui ont proliféré au cours du préstockage ainsi que des Penicillium (moisissures de stockage) qui ont rapidement prédominé. Après six mois de stockage, les espèces thermosensibles (Cladosporium cladosporioides, Cephalosporium acremonium, Fusarium roseum) ayant pratiquement disparu, la mycoflore est essentiellement constituée par des Penicillium et du Verticillium malthousei.

Les lots $\mathrm{F}$ et $\mathrm{F}$ ont été le siège d'une multiplication microbienne. C'est une raison suffisante pour qu'ils soient considérés comme suspects à cause d'une éventuelle production de métabolites toxiques, toujours possible dans ces conditions.

\section{CONCLUSIONS}

Des résultats obtenus, il ressort que les opérations de séchage ont eu une action dépressive et sélective sur la microflore des grains, assurant ainsi un assainissement des lots. Il est à noter que l'efficacité des traitements a été peu influencée par les températures de séchage. Les lots séchés immédiatement après la récolte sont les moins riches en microorganismes, donc les plus satisfaisants sur le plan hygiénique, d'autant qu'ils n'ont pas été le siège d'une prolifération microbienne après la récolte.

Les lots $\mathrm{E}$ et $\mathrm{F}$ doivent être considérés comme médiocres, d'une part ils possèdent une microflore assez abondante, d'autre part, les grains ont pu subir des dégradations d'ordre microbiologique au cours du préstockage. 


\section{SUMMARY}

\section{III. - DEVELOPMENT OF THE GRAIN MICROFLORA}

The results of enumerating the microbiological population during storages are expressed in total bacteria, coliform bacteria, and moulds.

The main values collected during the experiment demonstrate the comparative efficiency of the processing and drying conditions. It is also possible to draw conclusions about the control of the stability of the samples, and to appreciate the hygienic quality of the grains. The best samples are obtained by drying immediately after harvesting.

\section{RÉFÉRENCES BIBLIOGRAPHIQUES}

Christensen C. M., Kaufmann H. H., I965. Deterioration of stored grains by fungi. Ann. Rev. Phytopath., 3, 69-84.

Moreau C., r968. Moisissures toxiques dans l'alimentation, Lechevalier éd, Paris, $37 \mathrm{x}$.

Multon J. L., Poisson J., Guilbot A., I969. Les altérations du grain de blé après la récolte. Bull. Tech. Inf. Paris 244, I-18.

Poisson J., Saint-Lèbe L., Cahagnier B., 1964. Action de l'irradiation gamma sur la microflore de surface des grains. Rapport au C.E. A.

Porsson J., I967. Mise au point de chimie analytique organique, pharmaceutique et bromatologique, chapitre V, I27-148. Masson éd., Paris.

SEMENiUK G., I954. Microflora, in storage of cereal grains and their products, 77-151. Édité par l'A. A. C. C. Saint-Paul, Minnesota.

SpIcher G., I962. Die Ermittlung des Keimgehaltes von Getreideprodukten und ihre Problematik. Brot und Gebäck, 16, I24-I34. 